\title{
TGRS Observation of the Galactic Center Annihilation Line
}

B. J. Teegarden ${ }^{1}$, T. L. Cline, N. Gehrels, D. Palmer, R. Ramaty, H. Seifert, NASA/Goddard Space Flight Center

\author{
K. H. Hurley \\ University of California, Berkeley \\ D. A. Landis, N. W. Madden, D. Malone, R. Pehl \\ Lawrence Berkeley Laboratories \\ and
}

A. Owens

University of Leicester

Received —

Subtmitted to ApJL, 2/19/96

\footnotetext{
${ }^{1}$ On leave at Centre d'Etudes Spatiale des Rayonnements, Toulouse
} 


\begin{abstract}
The TGRS (Transient Gamma-Ray Spectrometer) experiment is a highresolution germanium detector launched on the WIND satellite on Nov. 1, 1994. Although primarily intended to study gamma-ray bursts and solar flares, TGRS also has the capability of studying slower transients (e.g. x-ray novae) and certain steady sources. We present here results on the narrow $511 \mathrm{keV}$ annihilation line from the general direction of the Galactic Center accumulated over the period Jan. 1995 through Oct. 1995. These results were obtained from the TGRS occultation mode, in which a lead absorber occults the Galactic Center region for $1 / 4$ of each spacecraft rotation, thus chopping the $511 \mathrm{keV}$ signal. The occulted region is a band in the sky of width $16^{\circ}$ that passes through the Galactic Center. We detect the narrow annihilation line from the galactic

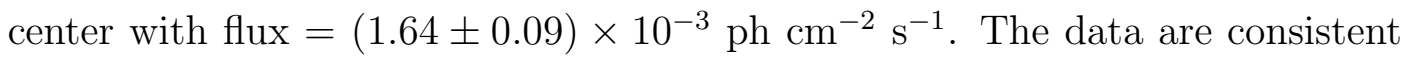
with a single point source at the galactic center, but a distributed source of extent up to $\sim 30^{\circ}$ cannot be ruled out. No evidence for temporal variability on time scales longer than 1 month was found.
\end{abstract}




\section{Introduction}

Electron-positron annihilation radiation is a valuable diagnostic for studies of a variety of different types of astrophysical sources. Positrons can be produced by hot pair plasmas in the vicinity of compact objects, by nucleosynthetic processes (both hydrostatic and explosive) and by cosmic-ray interactions in the interstellar medium. A narrow $511 \mathrm{keV}$ electron-positron annihilaton line ( $\sim 3 \mathrm{keV}$ FWHM) has been observed from the general direction of the Galactic Center (see Teegarden 1994 and Ramaty \& Lingenfelter 1995 for recent reviews). Data from a series of balloon observations in the late seventies and eighties suggested that the line was variable. These same instruments had a variety of different fields-of-view, and it was noted (see Teegarden 1994) that the detected flux varied systematically with the size of the field-of-view. This implied that there was also a diffuse steady component to the narrow $511 \mathrm{keV}$ emission. Subsequent satellite observations with SMM (Share et al. 1990) and CGRO/OSSE (Purcell et al. 1993, 1994) found no evidence for variability. CGRO/OSSE has now mapped the Galactic Center region with its $3^{\circ} \times 11^{\circ}$ collimator (Purcell et al. 1993,1994). The OSSE data can be well-represented by a two-component distribution consisting of a central bulge component of width $\sim 8^{\circ}$ combined with a disk-like component. $\sim 75 \%$ of the total flux is contained in the bulge component and $\sim 25 \%$ in the disk component.

The GRANAT/SIGMA experiment reported episodes of transient broad line emission, lasting typically a fraction of a day, from the source 1E1740.7-2942, which lies within $1^{\circ}$ of the Galactic Center (Bouchet et al. 1991; Sunyaev et al. 1991; Cordier et al. 1993). The line energy was $\sim 480 \mathrm{keV}$, and it was broadened to $\sim 130 \mathrm{keV}$. The features were interpreted as electron-positron annihilation radiation redshifted by the strong gravitational field of a black hole (Bouchet et al. 1991; Sunyaev et al. 1991). It has been suggested (Ramaty et al. 1992) that sources such as these can inject positrons into the ISM which 
can travel substantial distances before annihilating. An ensemble of such sources could be responsible for the diffuse narrow $511 \mathrm{keV}$ emission from the direction of the Galactic Center.

\section{Instrumentation}

TGRS (Transient Gamma-Ray Spectrometer) is an unshielded high-resolution 35- $\mathrm{cm}^{2}$ germanium detector passively cooled to a temperature of $85 \mathrm{~K}$ (for more detail see Owens et al. 1995). Its energy range is $\sim 20-8000 \mathrm{keV}$, and the energy resolution after launch was $\sim 2.7 \mathrm{keV} \mathrm{FWHM} \mathrm{at} 500 \mathrm{keV}$. TGRS was launched on the WIND spacecraft on 1 Nov. 1994. The primary purpose of the WIND mission is to sample conditions in interplanetary space in the vicinity of the earth's magnetosphere. Although in earth orbit, WIND spends the great majority of the time in interplanetary space. As a consequence the environment is generally quite stable (background levels, temperatures, etc.) which lends itself quite well to the long data accumulations necessary for the analysis described in this paper. A schematic view of the detector/occulter assembly is shown in Figure. 1. The main axis of the detector is coincident with the spin axis of the spacecraft, which is normal to the plane of the ecliptic. The $+\mathrm{Z}$ axis of the spacecraft (and the TGRS main field-of-view) is oriented towards the south ecliptic pole. This orientation was chosen so that the Large Magellanic Cloud (presumed location of the 5 Mar 1978 gamma-ray burst) is in the TGRS

field-of-view. The occulter is a 1-cm thick lead absorber located just outside of the radiative cooler in the plane of the detector, which subtends an angle of $90^{\circ}$ with respect to the detector. Additional structure and shielding (not shown in Figure 1) limit the low-energy threshold in the occultation mode to $\sim 40 \mathrm{keV}$. The on-board processor accumulates data in 4 commandable energy windows (64 channels ea.) synchronized with the spin of the spacecraft. One of these is centered on the $511 \mathrm{keV}$ annihilation line. Each spacecraft 
rotation (period $=3 \mathrm{~s}$.) is divided into 128 sectors. Due to spacecraft telemetry limitations one complete readout of all spin-synchronized data takes $\sim 2 \mathrm{hr}$, which defines the minimum time resolution in this mode.

Figure. 2 shows the region of the sky in galactic coordinates that is occulted (FWHM $=16^{\circ}$ ) by TGRS. The occulter has been offset by an angle of $5.5^{\circ}$ with respect to the spin plane to make the center of the occultation band coincide with the Galactic Center. The Crab also lies within the occultation region. Also shown in Figure 2 is a contour plot of the OSSE $511 \mathrm{keV}$ flux distribution (Purcell et al. 1994). As can be seen, the TGRS occulter modulates most of the flux in the central bulge component of the OSSE distribution.

\section{Observations}

Preliminary data are presented here for the period Jan.-Oct. 1995. The excellent stability of the instrument allows accumulations of long periods of data with no requirement for gain corrections. The $511 \mathrm{keV}$ background line has been used for gain calibration. Figure 3a shows the counting rate in a window $10 \mathrm{keV}$ wide centered on the $511 \mathrm{keV}$ line vs. the spin phase of the spacecraft, which is divided into 128 bins. The on-board accumulation is synchronized by a sun-sensor, and the data have been corrected for the sidereal variation of the phase. The solid line is the best fit to the data $\left(\chi^{2}=155,125\right.$ degrees of freedom, $\left.P\left(\chi^{2}, \nu\right)=0.036\right)$ for a single point source model in which the amplitude and position of the source are allowed to vary. The best-fit value for the source flux is $(1.64 \pm 0.09) \times 10^{-3} \mathrm{ph} \mathrm{cm}^{-2} \mathrm{~s}^{-1}$ and for the sector no. of the source is $95.3 \pm 0.4$. The

sector number corresponding to the Galactic Center is 94.9. To check for systematic effects that could mimic the signal from the Galactic Center a control region (516 - $539 \mathrm{keV})$ adjacent to the $511 \mathrm{keV}$ window was also examined. This is shown in Figure 3b. There is no evidence for any significant signal from the direction of the Galactic Center. A small 
residual in the sector range 15 - 40 is present due to hard x-ray emission from the Crab.

The data have been tested for the possible presence of a broadened source. Two broadened models (rectangular and guassian profiles in ecliptic longitude) were fit to the data. While the best fit values for the widths were non-zero $\left(21^{\circ}\right.$ in the rectangular case and $16^{\circ}$ in the gaussian case) the reduction in $\chi^{2}$ was not significant $\left(\Delta \chi^{2} \approx 2,124\right.$ degrees of freedom, for both models). The $90 \%$ confidence upper limit on the width of the rectangular model is $34^{\circ}$ and on the FWHM of the gaussian model is $30^{\circ}$. The TGRS data are therefore consistent with a point source at the Galactic Center, however, an extended source distribution with width up to $\sim 30^{\circ}$ cannot be ruled out..

Figure 4 shows the channel-by-channel spectrum in the vicinity of $511 \mathrm{keV}$ derived from the TGRS occultation data. The spectrum was calculated for an assumed point source at the Galactic Center. The data over the 300 day period were accumulated as a function of energy and spin phase. The spectra were derived as follows. Let $C_{i}\left(E_{j}\right)=$ no. of observed counts in the $\mathrm{i}^{\text {th }}$ spin sector and the $\mathrm{j}^{\text {th }}$ energy channel, $M_{i}=$ transmission of occulter for the $\mathrm{i}^{\text {th }}$ spin sector, $S\left(E_{j}\right)=$ source counts in the $\mathrm{j}^{\text {th }}$ energy channel and $B\left(E_{j}\right)=$ background counts in the $\mathrm{j}^{\text {th }}$ energy channel. Over the limited band of energy in question (506 - $516 \mathrm{keV}) M_{i}$ is taken to be independent of energy. The counts in each sector and energy bin are then given by:

$$
C_{i}\left(E_{j}\right)=M_{i} S\left(E_{j}\right)+B\left(E_{j}\right)
$$

The source spectrum is derived by linear regression on $C\left(E_{j}\right)$ of $\mathrm{M}$, with a constant term. The coefficient of $\mathrm{M}$ gives the source flux and the constant $\mathrm{B}$ gives the background at $E_{j}$. This is equivalent to the previously discussed model fit with the source location held fixed (at the best fit value) and the energy range restricted to a single energy channel.

The spectrum in Figure 4 displays a narrow line at $511 \mathrm{keV}$. A gaussian fit to the line yields a flux of $(1.64 \pm 0.08) \times 10^{-3} \mathrm{ph} \mathrm{cm}^{-2} \mathrm{~s}^{-1}$ in excellent agreement with that derived 
from the data of Figure 3. There is a suggestion of line broadening. However, no gain shift corrections have been applied to the data, and there may, as a reult, be some broadening beyond the normal instrumental resolution. Further careful analysis will be necessary to remove this effect (and possibly others) to obtain a reliable absolute value for the line width.

Figure 5 is a time history of the intensity of the Galactic Center $511 \mathrm{keV}$ line plotted on a monthly basis. The data points were derived by determining the best-fit values for the flux using the TGRS spin sector data such as shown in Figure 3. A single point source was assumed, and both the flux and position were allowed to vary. In all cases the best-fit position was consistent with Galactic Center. The data are consistent with a constant flux with a $\chi^{2}$ probability of $\sim 17 \%$.

\section{Discussion}

Through its occultation mode TGRS has detected a significant $(\sim 20 \sigma)$ flux of $511 \mathrm{keV}$ emission from the direction of the Galactic Center. The region observed corresponds to a band in the sky of FWHM $16^{\circ}$ offset by $-5.5^{\circ}$ from the ecliptic plane, which passes through the Galactic Center (see Figure 2). Purcell et al. (1994) have fit the OSSE $511 \mathrm{keV}$ data with a diffuse distribution consisting of a central bulge component and a disk component (see Figure 2). The respective fluxes in these two components are $1.7 \times 10^{-3} \mathrm{ph} \mathrm{cm}^{-2} \mathrm{~s}^{-1}$ and $0.5 \times 10^{-3} \mathrm{ph} \mathrm{cm}^{-2} \mathrm{~s}^{-1}$. If one convolves the OSSE model distribution with the TGRS occulter modulation function (for the occulter centered at the Galactic Center) one obtains an occulted flux of $1.5 \times 10^{-3} \mathrm{ph} \mathrm{cm}^{-2} \mathrm{~s}^{-1}$. This is to be compared with the measured TGRS occulted flux of $(1.64 \pm 0.09) \times 10^{-3} \mathrm{ph} \mathrm{cm}^{-2} \mathrm{~s}^{-1}$. The TGRS and OSSE absolute flux measurements are therefore mutually consistent at approximately the $1 \sigma$ level.

The TGRS data can be well fit by a point source at an ecliptic longitude of $268.0 \pm 1.2^{\circ}$ 
$(\mathrm{J} 2000)$ which is $1.0 \sigma$ from the Galactic Center ( ecliptic longitude $=266.8^{\circ}$ ). The ecliptic longitude of the source $1 \mathrm{E} 1740.7-2942$ is $266.4^{\circ}$ or within $1.3 \sigma$ of the best-fit TGRS position. The TGRS $511 \mathrm{keV}$ source position is therefore consistent with both the Galactic Center and the 1E1740.7-2942 source. Because of the proximity of the TGRS occulter to the germanium detector, and the finite size of the detector itself, the effective TGRS point spread function is rather large (see Figure 3). It is therefore not possible to make a definitive statement on the spatial extent of the source. The data were fit with two different broadened source distributions, rectangular and gaussian. While the best-fit values for the width were non-zero ( $21^{\circ}$ for rectangular and $16^{\circ} \mathrm{FWHM}$ for the gaussian distribution), the change in $\chi^{2}$ was not significant $\left(\Delta \chi^{2} \simeq 2\right.$ in both cases). The $90 \%$ confidence upper limits on the widths were $34^{\circ}$ for the rectangular model and $30^{\circ} \mathrm{FWHM}$ for the gaussian model.

The historical evidence for variability in the Galactic Center $511 \mathrm{keV}$ flux comes primarily from a series of balloon flights from the late 1970's to the early 1990's. Two flights in 1981 (Leventhal et al. 1982; Paciesas et al. 1982) and one in 1985 (Leventhal et al. 1986) found no detectable narrow $511 \mathrm{keV}$ line flux. These instruments had fields-of-view in the $15-20^{\circ}$ range and sufficient sensitivity to detect emission at the levels implied by the OSSE data. Teegarden (1994) showed that these data were inconsistent with a constant flux at the $1 \%$ level. However, the error estimates generally did not include systematic effects, which could reduce the significance of the time variation. Initial results from HEAO-3 suggested a variation in the Galactic Center $511 \mathrm{keV}$ flux between the fall of 1979 and the spring of 1980 (Riegler et al. 1981). However, a subsequent re-analysis by Mahoney et al. (1993) reduced the variation to an insignificant value. None of the other satellite observations (SMM, OSSE, TGRS) have found any evidence for temporal variability. The available body of evidence weighs most heavily in favor of a constant diffuse source for the narrow Galactic Center annihilation radiation, although a variable point source at a level $<2 \times 10^{-4} \mathrm{ph} \mathrm{cm}^{-2}$ cannot be ruled out (Ramaty et al. 1994; Purcell et al. 1994). 
The authors would particularly like to recognize the efforts of the ISTP/WIND project staff (J. Hrastar, project manager, W. Anselm, instrument coordinator). Particular credit is to be given to D. A. Sheppard, D. Stilwell, A. Post, S. Matthias, C. Cork and P. Luke for the development of the flight hardware. Special recognition is due to S. Bansal and T. Sheets for the development of the TGRS data processing software. The authors wish to thank Wm. Purcell for many helpful comments. 


\section{REFERENCES}

Bouchet, L. et al. 1991, ApJ, 383, L45

Cordier, B. et al. 1993, A\&A, 275, L1

Leventhal, M. L. et al. 1982, ApJ, 260, L1

Leventhal, M. L. et al. 1986, ApJ, 302, 459

Mahoney, W. et al. 1993, ApJS, 92, 387

Owens, A. 1995, Spa. Sci. Rev., 71, 273

Paciesas, W. et al. 1982, ApJ, 260, L7

Purcell, W.R. et al. 1993, ApJ, 413, L85

Purcell, W.R. et al. 1994, in Proc. Second Compton Symposium (College Park), 403

Ramaty, R. et al. 1992, ApJ,392, L63

Ramaty, R. et al. 1994, ApJS, 92, 393

Ramaty R. \& Lingenfelter,R.E. 1995, in The Analysis of Emission Lines (Cambridge), in press.

Riegler, G. R. et al. 1981, ApJ, 248, L13

Share, G. J. et al. 1990, ApJ, 358, L45

Sunyaev, R. et al. 1991, ApJ, 383, L49

Teegarden, B. J. 1994, ApJS, 92, 363 
Fig. 1.- Schematic view of the TGRS detector/occulter. The spacecraft spin axis is coincident with the detector axis and normal to the ecliptic plane. The occulter subtends an angle of $90^{\circ} \times 16^{\circ}(\mathrm{FWHM})$ with respect to the detector.

Fig. 2.- Band of the sky modulated by the TGRS occulter. Also shown is a contour plot of the $511 \mathrm{keV}$ annihilation radiation as measured by OSSE. Contours are logarithmically spaced with a ratio between contours of 3.5. The location of the Crab, which lies within the occulation band, is also shown.

Fig. 3.- Counting rate as a function of spacecraft spin sector (128 sectors/spin). Data are accumulated between 1 Jan. 1995 and 27 Oct. 1995 (300 days). (a) Energy range 506 - 516 $\mathrm{keV}$. Solid line is best-fit single point source model. The location is consistent with Galactic Center. (b) Energy range 516-539 keV. No evidence for a significant source at the Galactic Center is present.

Fig. 4.- TGRS spectrum in vicinity of $511 \mathrm{keV}$ line. Data accumulated from 1 Jan. 1995 to 27 Oct. 1995. Energy of line is consistent with $511 \mathrm{keV}$.

Fig. 5.- Time history of $511 \mathrm{keV}$ line intensity during 1995. 


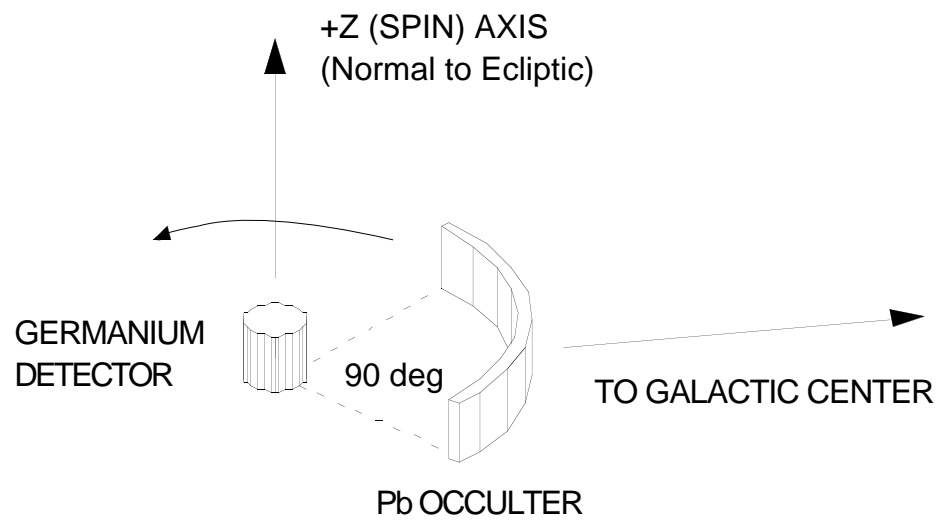




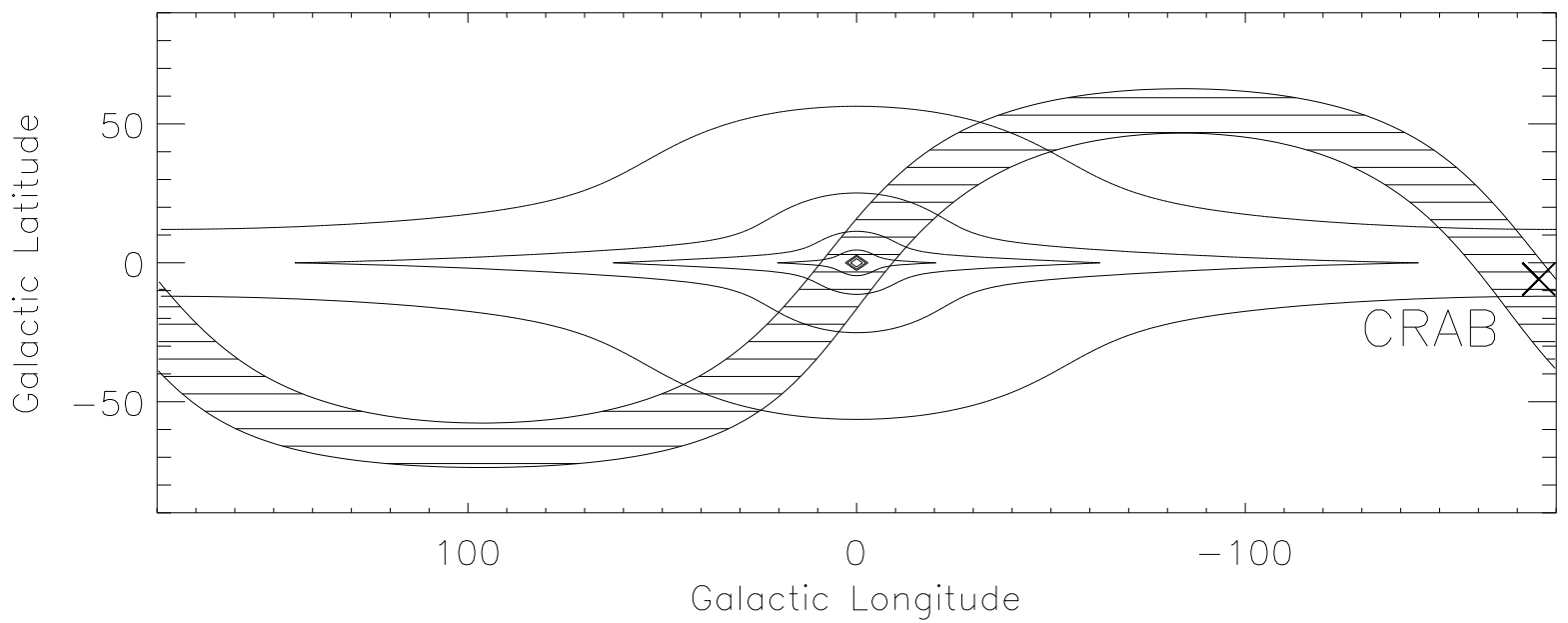



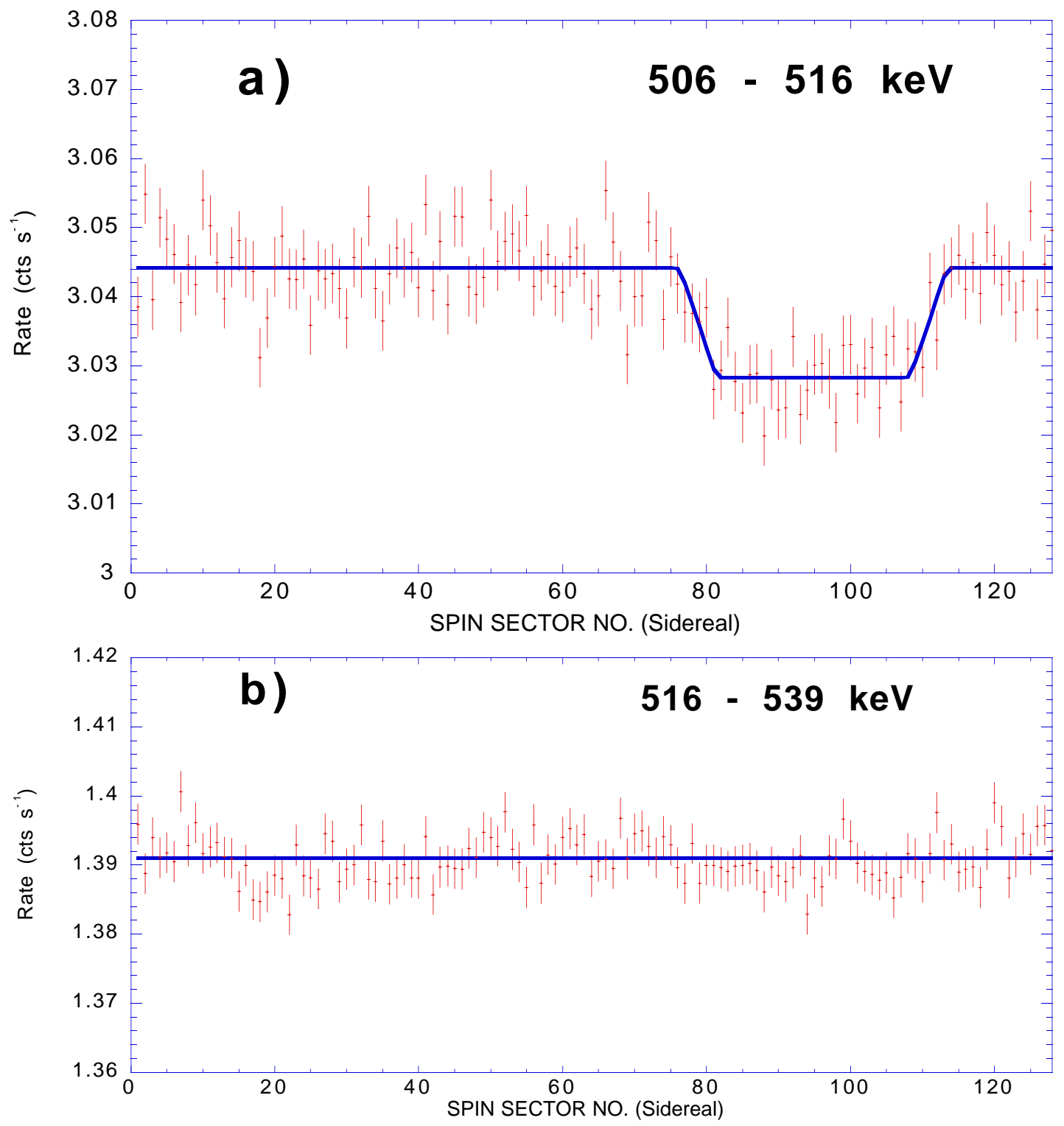


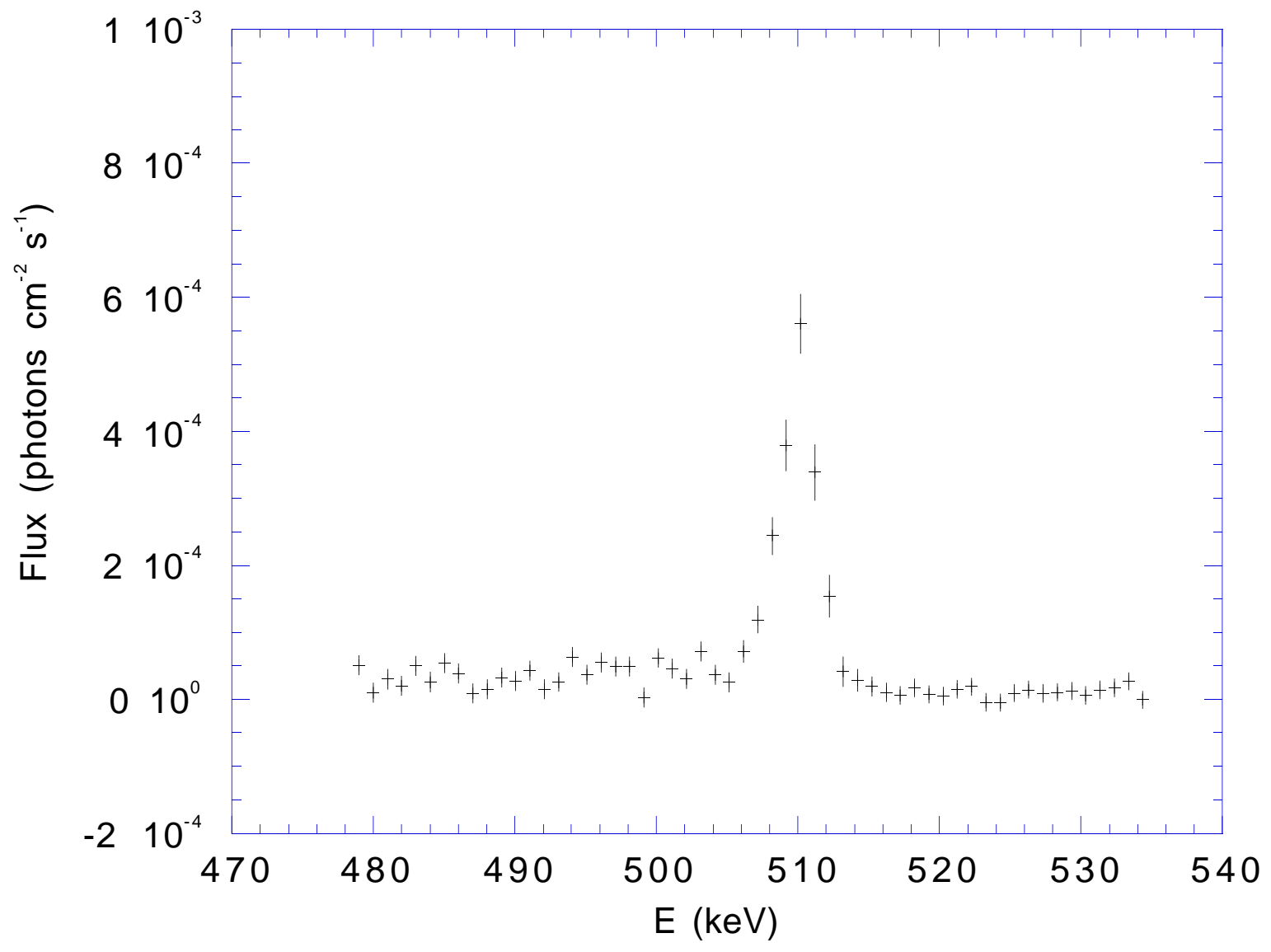




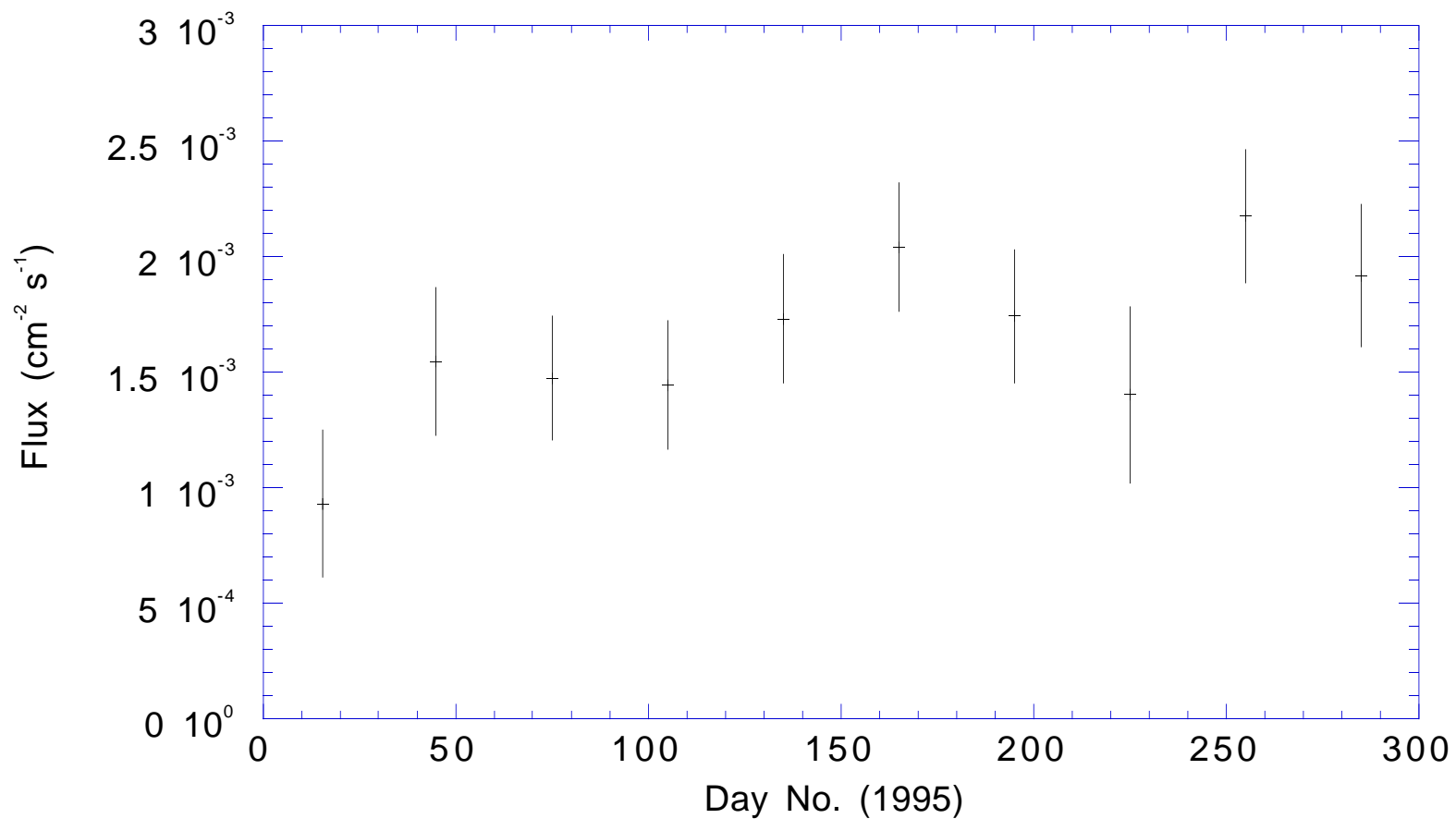

